\title{
The Ratio of $\sigma_{L} / \sigma_{T}$ in DIS at Low $x$
}

\author{
Dieter Schildknecht ${ }^{1,2}$ \\ 1- Fakultät für Physik, Universität Bielefeld, Universitätsstrasse 25, D-33615 Bielefeld, Germany \\ 2- MPI für Physik, München, Föhringer Ring 6, D-80805 München, Germany
}

\begin{abstract}
Assuming helicity independence for $q \bar{q}$ scattering in the color-dipole picture, or, equivalently proportionality of sea quark and gluon distributions, we find $R\left(W^{2}, Q^{2}\right) \cong 0.5$ at large $Q^{2}$, where $R\left(W^{2}, Q^{2}\right)$ denotes the ratio of the longitudinal and transverse photoabsorption cross sections. The forthcoming direct measurements of $R\left(W^{2}, Q^{2}\right)$ allow one to test the underlying hypotheses.
\end{abstract}

This is a brief summary of my talk at DIS 2007. We also refer to the slides of the talk, available under http://indico.cern.ch/confAuthorIndex.py? confId=9499. It was recently noted [2] that the dipole picture [3] of deep inelastic scattering at low $x \cong Q^{2} / W^{2} \leq 0.1$,

$$
\sigma_{\gamma_{L, T}^{*} p}\left(W^{2}, Q^{2}\right)=\sum_{q} \int d^{2} r_{\perp} \omega_{L, T}^{(q)}\left(Q r_{\perp}, Q^{2}, m_{q}^{2}\right) \sigma_{(q \bar{q}) p}\left(r_{\perp}^{2}, W^{2}\right),
$$

allows one to derive an upper bound on the ratio of the cross sections induced by longitudinal and transverse photons,

$$
R\left(W^{2}, Q^{2}\right)=\frac{\sigma_{\gamma_{L}^{*} p}\left(W^{2}, Q^{2}\right)}{\sigma_{\gamma_{T}^{*} p}\left(W^{2}, Q^{2}\right)} \leq \max _{r_{\perp}, q} \frac{\omega_{L}^{(q)}\left(Q r_{\perp}, Q^{2}, m_{q}^{2}\right)}{\omega_{T}^{(q)}\left(Q r_{\perp}, Q^{2}, m_{q}^{2}\right)}=0.37 .
$$

Since the photon fluctuates into on-shell $q \bar{q}$ states

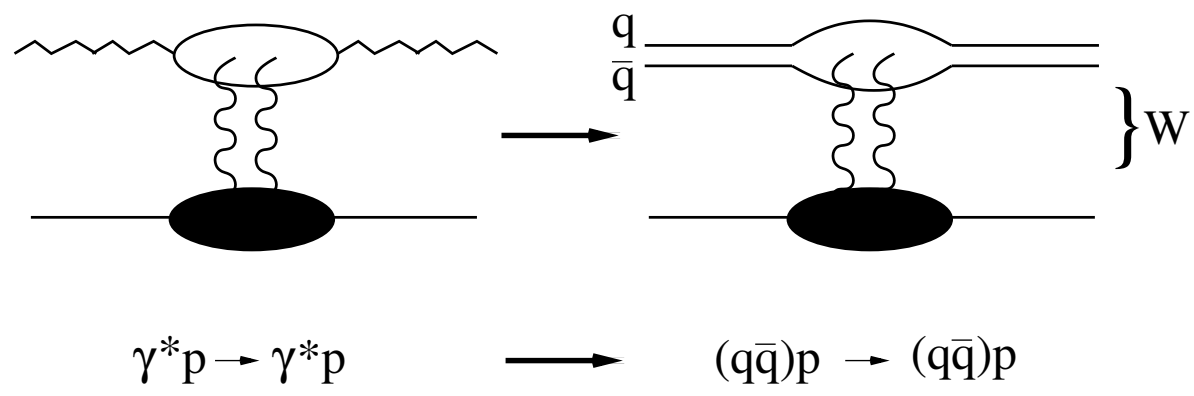

the $q \bar{q}$-scattering process entering the virtual Compton-forward-scattering amplitude is identical to the $q \bar{q}$-scattering process of on-shell $q \bar{q}$ states. Accordingly, as indicated in (1), the cross section factorizes into a $Q^{2}$-dependent probability density and a $W^{2}$-dependent (rather than $x$-dependent) dipole cross section. The relevance of the energy $W$ as the dynamical variable in the low- $x$ diffraction region may be traced back to the representation of low$x$ deep inelastic scattering in terms of generalized vector dominance [4] some thirty-five years ago. For the connection between the dipole picture and generalized vector dominance compare also refs. 4 and 5 and the recent review in 6 . The dependence on $W$ rather than $x$ on the right-hand side in (1) was recently stressed by Ewerz and Nachtmann [2] in their very elaborate and explicit treatment of the foundations of the dipole picture. 

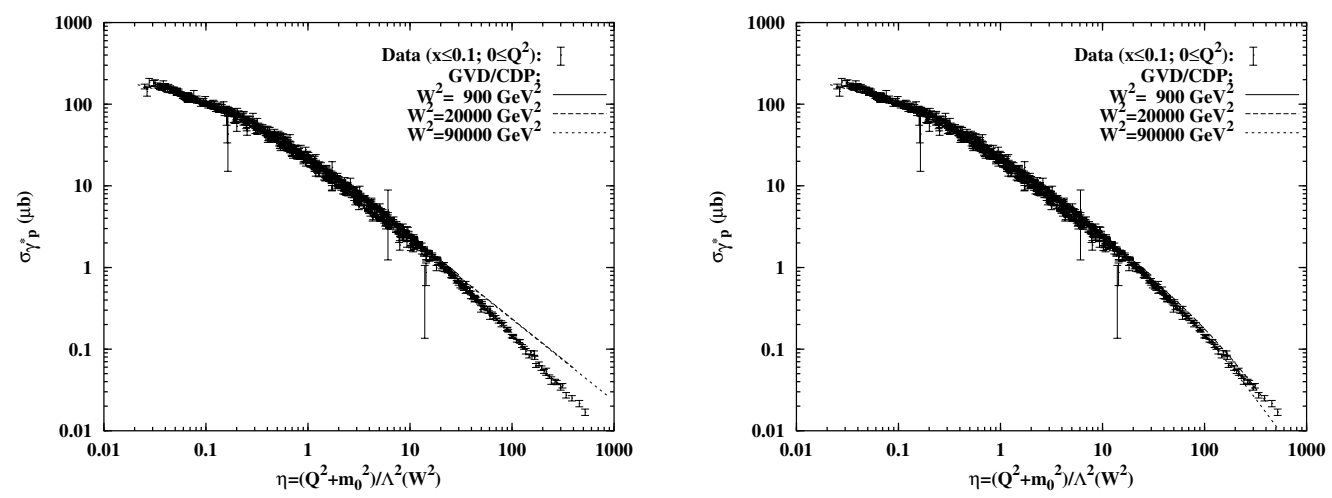

Figure 1: The total photoabsorption cross section for $m_{1}^{2}=\infty$ and $m_{1}^{2}=484 \mathrm{GeV}^{2}$.

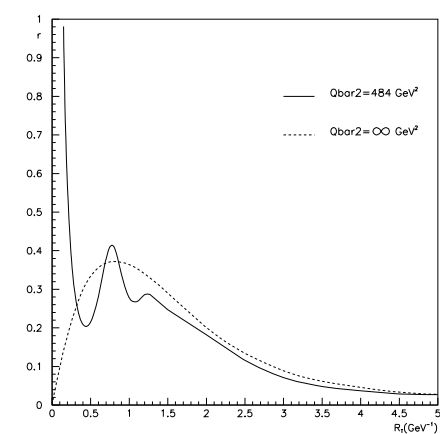

Figure 2: The ratio (7)

Since the available energy, $W$, is finite, the mass, $M_{q \bar{q}}$, of the contributing color dipoles must be bounded $[8,2]$,

$$
M_{q \bar{q}}^{2}=\frac{\vec{k}_{\perp}^{2}}{z(1-z)} \leq m_{1}^{2} \equiv \bar{Q}^{2}<<W^{2}
$$

The bound $\bar{Q}^{2}$ must be identified with the upper limit of the diffractively produced masses that is expected to be substantially below the available energy. In our representation of the HERA data, we used a value of [8]

$$
\bar{Q}^{2} \equiv m_{1}^{2}=(22 G e V)^{2},
$$

that was abstracted from the effective upper end of the diffractive mass spectrum observed at HERA. In Figure 1, we show that the introduction of the bound [8] (5) extends the range of validity of the representation of the cross section in terms of the scaling variable $[9,6]$

$$
\eta=\frac{Q^{2}+m_{0}^{2}}{\Lambda_{\mathrm{sat}}^{2}\left(W^{2}\right)}
$$

to the region of large values of $\eta$. Since $\bar{Q}^{2}$ is determined by the upper limit of diffractively produced masses, $\bar{Q}^{2}$ increases slowly with increasing energy. To adopt a constant value for the HERA energy range must be considered as an approximation.

We have analyzed[10] the effect of the restriction $(4,5)$ on the ratio $R\left(W^{2}, Q^{2}\right)$ in (2). The probability density to find a dipole of size $r_{\perp}$ in the (virtual) photon now becomes dependent on $\bar{Q}^{2}$. The ratio of the probability densities in (2), for finite $\bar{Q}^{2}$ diverges in the limit of small dipoles, $r_{\perp} \rightarrow 0$,

$$
r^{(q)}\left(Q r_{\perp}, \frac{Q^{2}}{\bar{Q}^{2}}\right)=\frac{\omega_{L}^{(q)}\left(Q r_{\perp}, Q^{2}, \bar{Q}^{2}\right)}{\omega_{T}^{(q)}\left(Q r_{\perp}, Q^{2}, \bar{Q}^{2}\right)} \stackrel{r_{\perp} \rightarrow 0}{\sim} \begin{cases}Q^{2} r_{\perp}^{2} \rightarrow 0 & , \text { for } \bar{Q}^{2} \rightarrow \infty \\ \frac{1}{r_{\perp}^{2} Q^{2}} \rightarrow \infty & , \text { for } \bar{Q}^{2} \text { finite }\end{cases}
$$

and the bound (2) turns into the trivial statement

$$
0 \leq R\left(W^{2}, Q^{2}\right)<\infty
$$


i.e. the derivation of an upper limit for $R\left(W^{2}, Q^{2}\right)$ fails, once a finite value for $\bar{Q}^{2}$ is adopted. Compare Figure 2 for the ratio of the probability densities, where for illustration the value (5) for $\bar{Q}^{2}$ is used.

Actually, the representation (1) of the dipole picture must be applied in conjunction with color transparency[3]

$$
\begin{aligned}
\sigma_{(q \bar{q}) p}\left(r_{\perp}^{2}, W^{2}\right) & =\int d^{2} \vec{l}_{\perp} \tilde{\sigma}_{(q \bar{q}) p}\left(\vec{l}_{\perp}^{2}, W^{2}\right)\left(1-e^{-i \vec{l}_{\perp} \vec{r}_{\perp}}\right) \\
& \simeq \vec{r}_{\perp}^{2} \frac{\pi}{4} \int d \vec{l}_{\perp}^{2} \vec{l}_{\perp}^{2} \tilde{\sigma}_{(q \bar{q}) p}\left(\vec{l}_{\perp}^{2}, W^{2}\right), \quad \text { for } \vec{r}_{\perp}^{2} \rightarrow 0 .
\end{aligned}
$$

Here, $\vec{l}_{\perp}$ denotes the transverse momentum of the gluon absorbed by the $q \bar{q}$ pair in the forward-scattering amplitude, where two gluons of opposite transverse momentum couple to the $q \bar{q}$ pair. Since both, transitions $M_{q \bar{q}} \rightarrow M_{q \bar{q}}$ as well as $M_{q \bar{q}} \rightarrow M_{q \bar{q}}^{\prime}$, occur, the restriction (4) is to be supplemented by

$$
M_{q \bar{q}}^{\prime 2}=\frac{\left(\vec{k}_{\perp}+\vec{l}_{\perp}\right)^{2}}{z(1-z)}<\bar{Q}^{2} .
$$

Noting that the momentum of the gluon is entirely independent of the transverse momentum of the quarks, $k_{\perp}$, restrictions (4) and (10) together require

$$
\vec{l}_{\perp}^{\prime 2}=\frac{\vec{l}_{\perp}^{2}}{z(1-z)}<<\bar{Q}^{2}
$$

i.e. the effective change in mass of the $q \bar{q}$ state by gluon absorption must be much smaller than the upper bound $\bar{Q}^{2}$, where $Q^{2}=m_{1}^{2} \simeq(22 G e V)^{2}$ at HERA.

In order to investigate the effect of the restrictions (4) and (11) on $R\left(W^{2}, Q^{2}\right)$, we appropriately start[10] with the limit of $\bar{Q}^{2}$ large compared with the effective value of the gluon transverse momentum, that is with the limit of $\bar{Q}^{2} \rightarrow \infty$. For $Q^{2}$ large compared with the effective value of $\vec{l}_{\perp}^{\prime 2}$, i.e. $\left.Q^{2}>><\vec{l}_{\perp}^{\prime 2}\right\rangle$, where $\left\langle\vec{l}_{\perp}^{\prime 2}\right\rangle$ is proportional to the "saturation scale" $\Lambda_{\text {sat }}^{2}\left(W^{2}\right)$, we find

$$
R\left(W^{2}, Q^{2}\right)=\underbrace{\frac{\int d y y^{3} K_{0}^{2}(y)}{\int d y y^{3} K_{1}^{2}(y)}}_{\frac{1}{2}} \cdot \frac{\int d \vec{l}^{\prime 2} \vec{l}_{\perp}^{\prime 2} \bar{\sigma}_{(q \bar{q})_{L}^{J=1} p}\left(\vec{l}_{\perp}^{\prime 2}, W^{2}\right)}{\int d \vec{l}^{\prime 2} \vec{l}_{\perp}^{\prime 2} \bar{\sigma}_{(q \bar{q})_{T}^{J=1} p}\left(\vec{l}_{\perp}^{\prime 2}, W^{2}\right)} .
$$

The ratio of the integrals over modified Bessel functions in (12) yields $1 / 2$. Note that the right-hand side in (12) depends on the ratio of the $q \bar{q}$ absorption cross sections for longitudinally and transversely polarized $(q \bar{q})^{J=1}$ (vector) states. Adopting the assumption of helicity independence $[6,8]$, i.e. equality of the first moment of the scattering amplitudes for longitudinal and transverse polarisation, we have from (12)

$$
R\left(W^{2}, Q^{2}\right)=0.5 .
$$

We summarize: With color transparency (two gluons coupled to $q \bar{q}$ ) and the hypothesis of helicity independence, we have $R\left(W^{2}, Q^{2}\right)=0.5$ at large $Q^{2}$. A preliminary investigation[10] indicates no substantial change of this result for $\bar{Q}^{2}$ finite. 
The hypothesis of helicity independence, at large $Q^{2}$ may be expressed in terms of a proportionality[11] of sea quark and gluon distributions. With the constant of proportionality, $\rho$, we then have

$$
R\left(W^{2}, Q^{2}>>\Lambda_{\text {sat }}^{2}\left(W^{2}\right)\right)=\frac{1}{2 \rho},
$$

where $\rho=1$ corresponds to (13). Applying the evolution equation at low $x$, and large $Q^{2}$, one finds $[11,12]$ a correlation between $\rho$ and the exponent in the $W^{2}$ dependence of the saturation scale,

given by

$$
\Lambda_{\text {sat }}^{2}\left(W^{2}\right)=\text { const. }\left(\frac{W^{2}}{1 G e V^{2}}\right)^{C_{2}}
$$

$$
(2 \rho+1) C_{2}^{\text {theor. }} 2^{C_{2}^{\text {theor. }}}=1
$$

Compare Table 1.

\begin{tabular}{|c|c|c|c|c|}
\hline \multicolumn{1}{c|}{} & $C_{2}^{\text {theor. }}$ & $\alpha_{s} \cdot$ glue & $\sigma_{\gamma_{L}^{*}} / \sigma_{\gamma_{T}^{*}}$ & $F_{2}\left(\frac{Q^{2}}{x}\right)$ \\
\hline$\rightarrow \infty$ & 0 & $\ll$ sea & 0 & $\left(Q^{2} / x\right)^{0}=$ const. \\
1 & 0.276 & $\approx$ sea & $\sim \frac{1}{2}$ & $\left(Q^{2} / x\right)^{0.276}$ \\
0 & 0.65 & $>$ sea & $\infty$ & $\left(Q^{2} / x\right)^{0.65}$ \\
\hline
\end{tabular}

The coincidence of the theoretical value of $C_{2}^{\text {theor. }}$ with the fit $[6]$ to the experimental data, $C_{2}^{\exp }=0.27 \pm$ 0.1 , supports helicity independence with $\rho=1$, i.e. $R\left(W^{2}, Q^{2}\right)=0.5$ at large $Q^{2}$. Measurements of $R\left(W^{2}, Q^{2}\right)$

Table 1: Results for $C_{2}^{\text {theor. }}$ for different values of $\rho$ allow one to directly test the limits of the assumed proportionality of sea and gluon distributions that is equivalent to helicity independence and correlated with the rise of $F_{2}\left(W^{2}=Q^{2} / x\right)$ as a function of $x$ at fixed $Q^{2}$.

\section{Acknowledgments}

Many thanks to Kuroda-san for a fruitful collaboration.

\section{References}

[1] Slides: http://indico. cern. ch/contributionDisplay.py?contribId=64\&sessionId=7\&conf Id=9499

[2] C. Ewerz and O. Nachtmann, arXiv:hep-ph/0404254, hep-ph/0604087, hep-ph/0511051.

[3] N.N. Nikolaev and B.G. Zakharov, Z. Phys. C49 607 (1991).

[4] J.J. Sakurai and D. Schildknecht, Phys. Lett. B40 121 (1972); B. Gorczyca and D. Schildknecht, Phys. Lett. B47 71 (1973); R. Devenish and D. Schildknecht, Phys. Rev. D19 93 (1976).

[5] G. Cvetic, D. Schildknecht, A. Shoshi, Eur. Phys. J. C13 301 (2000); Acta Physica Polonica B30 3265 (1999).

[6] D. Schildknecht, B. Surrow, M. Tentyukov, Phys. Lett. B499 116 (2001); G. Cvetic, D. Schildknecht, B. Surrow, M. Tentyukov, Eur. Phys. J. C20 77 (2001); D. Schildknecht, B. Surrow, M. Tentyukov, Mod. Phys. Lett. A16 1829 (2001).

[7] D. Schildknecht, in Proc. PHOTON2005, Acta Physica Polonica B37 (2006) 595.

[8] M. Kuroda and D. Schildknecht, Phys. Rev. D66 094005 (2002).

[9] D. Schildknecht, in Diffraction 2000, Nucl. Phys. B (Proc. Suppl.) 99121 (2001); D. Schildknecht, in: G. Bruni et al. (Eds.), DIS2001, Bologna, Italy, World Scientific, Singapore, p. 798 (2002).

[10] M. Kuroda and D. Schildknecht, in preparation.

[11] M. Kuroda and D. Schildknecht, Phys. Lett. B618 84 (2005).

[12] D. Schildknecht, in 41st Rencontres de Moriond, March 2006, arXiV:hep-ph/0607031. 